\title{
Gas gangrene in a diabetic after intramuscular injection
}

\author{
C.J. Kershaw and C.J.K. Bulstrode
}

Accident Service, John Radcliffe Hospital, Headington, Oxford, UK.

\begin{abstract}
Summary: A 47 year old male insulin-dependent diabetic developed two synchronous lifethreatening Clostridium perfringens infections under unusual circumstances. Numerous sterile abscesses were also present, being the result of regular pentazocine intramuscular injections.

Extensive surgical debridement and parenteral antibiotic therapy proved effective in treating the septicaemia and the large soft tissue abscesses in the buttock and thigh. The patient made an excellent functional recovery.

The pathogenesis of gas gangrene after injection is discussed with particular reference to the diabetic patient.
\end{abstract}

\section{Introduction}

Infections in diabetics are common, and it is well recognized that apparently insignificant infections can progress rapidly to become life-threatening. Gas gangrene is also well recognized in diabetics but is usually a consequence of lower limb surgery. ${ }^{1}$ Gas forming soft tissue infections in diabetics are usually non-clostridial, caused by aerobic and anaerobic streptococci and Escherichia coli. ${ }^{2}$ A case is presented of a diabetic who developed two Clostridium perfringens infections under unusual circumstances.

\section{Case report}

A 47 year old insulin dependent diabetic was admitted with a 6-day history of painful, tender swellings in his left thigh and buttock. Five days before he had consulted his family practitioner and had been prescribed oral flucloxacillin. He had felt unwell for the previous 24 hours with thirst and polyuria.

He had had a peptic ulcer treated by Polya gastrectomy in 1962, with full symptomatic relief. From 1982 until 1984 he had twelve hospital admissions due to episodes of severe abdominal pain. A laparotomy was finally performed revealing adhesions, which were divided, and chronic pancreatitis. His pain was unaffected and in late 1984 he underwent partial pancreatectomy after several unsuccessful attempts at coeliac axis nerve blockade. He subsequently developed insulin-dependent

Correspondence: C.J. Kershaw, F.R.C.S.

Accepted: 11 May 1988 diabetes. His pain was only partly improved. Numerous analgesics were tried, but only regular intramuscular pentazocine, injected into both thighs and buttocks, was effective, supplemented by oral dihydrocodeine. There were conflicting opinions from both surgeons and psychiatrists as to whether he had Munchausen's syndrome. His insulin waso always injected into the deltoid region. Syringes and? needles were re-used, but there was no interchanging between those used for insulin injection and those used for pentazocine injection. He required pancreatic supplements with food, but was well nourished. His diabetes was stable with only occasional glycosuria.

On admission to hospital he looked unwell, with a pyrexia of $39^{\circ} \mathrm{C}$ associated with a tachycardia and tachypnoea. He had non-tender fluctuant swellings over both deltoid regions and the right mid-thigh. There were large, tender, tympanic, fluctuant swellings over the anterior aspect of the left thigh and the outer half of the left buttock. The overlying skin was red and indurated. Haematological investigations revealed white cell count $21 \times 10^{9} \mathrm{l}$; blood glucose $22.8 \mathrm{mmol} / \mathrm{l}$. Radiographs of the left leg showed two large gas filled cavities, one in the buttock and one in the quadriceps. Radiographs of the other swellings showed no evidence of gas.

He was resuscitated and under general anaesthesia the three non-tender swellings were aspirated yielding thick, odourless, brown pus. Microscopy revealed only pus cells and subsequent culture was sterile. The two tender swellings were incised. The buttock abscess was subcutaneous, with no muscle involvement, and contained a large volume of foulsmelling gas and $500 \mathrm{ml}$ of brown pus. The cavity

(C) The Fellowship of Postgraduate Medicine, 1988 
was debrided and packed with Eusol and paraffin gauze. The thigh abscess also contained considerable volumes of malodorous gas and pus, but with extensive muscle necrosis and gas bubbles in the muscle. Rectus femoris was most affected and was completely excised. Considerable proportions of the vasti and subcutaneous tissue also required excision. The wound was left open. Microscopy of pus from both these abscesses revealed large quantities of Gram positive rods. Culture confirmed a pure growth of Clostridium perfringens sensitive to benzyl penicillin and metronidazole. Blood cultures were similarly positive. Parenteral therapy with benzyl penicillin (4 megaunits 6-hourly) and metronidazole (1 gram 8-hourly) was commenced. Polyvalent clostridial antitoxin was given. Postoperatively he required ventilation for a short period but then progressed remarkably well. Regular wound dressings under general anaesthesia were performed. Both wounds granulated well, only a small area of the thigh wound requiring split-skin grafting to obtain final healing.

\section{Discussion}

Pyogenic infections at injection sites in diabetics are not uncommon, but to our knowledge gas gangrene has not been reported. Gas gangrene has occurred

\section{References}

1. Kahn, $O$. The incidence and significance of gas gangrene in a diabetic population. Angiology 1974, 25: $462-466$.

2. Wills, M.R. \& Reece, M.W. Non-clostridial gas infections in diabetes mellitus. $\mathrm{Br}$ Med $J$ 1960, 2: 566-568.

3. Harvey, P.W. \& Purnell, G.V. Fatal case of gas gangrene associated with intramuscular injection. $\mathrm{Br}$ Med J 1968, 1: 744-746. after intramuscular injection of adrenaline, this practice now being condemned. ${ }^{3}$

Clostridia thrive in damaged tissues where the vascularity is impaired and low oxygen tensions prevail. Increasing tissue tension as oedema develops and gas accumulates further compromises the tissues' blood supply.

Diabetics are prone to peripheral neuropathy and microangiopathy, both of which can delay tissue healing and encourage opportunist infections. Phagocytosis and intracellular bactericidal activity have been shown to be affected by the control of glycaemia, and there may be some immunological impairment which is independent of glycaemic control. ${ }^{4}$

The presence of sterile abscesses at other injection sites suggested that clostridial spores had infected previously sterile abscesses. The presence of pre-existing tissue necrosis in a hyperglycaemic environment would provide excellent conditions for bacterial proliferation. It is noteworthy that insulin in vials contains a strong antiseptic whereas pentazocine does not. This may have prevented gas gangrene infection of the deltoid abscesses.

Radical excision of all necrotic tissue and adequate doses of parenteral antibiotics remain the mainstay of treatment of this dangerous condition. ${ }^{5}$ To reduce the diabetic's predisposition to infection, better metabolic control is essential.

4. Larkin, J.G., Frier, B.M. \& Ireland, J.T. Diabetes mellitus and infection. Postgrad Med $J$ 1985, 61: 233-237.

5. Hart, G.B., Lamb, R.C. \& Strauss, M.B. Gas gangrene: a collective review. J Trauma 1983, 23: 991-1000. 